\title{
The key role of vitamin A in spermatogenesis
}

\author{
Cathryn A. Hogarth and Michael D. Griswold
}

School of Molecular Biosciences, Washington State University, Pullman.

\begin{abstract}
Spermatogenesis in adult mammals is highly organized, with the goal being continual sperm production. Vertebrate testes are arranged into recurring cellular associations that vary with time and distance along the tubule. These changes over time and distance are designated the cycle of the seminiferous epithelium and the spermatogenic wave, respectively. In this Review, we briefly outline the roles that follicle-stimulating hormone (FSH) and testosterone play in regulating spermatogenesis and describe our current understanding of how vitamin $\mathrm{A}$ regulates germ cell differentiation and how it may lead to the generation of both the cycle of the seminiferous epithelium and the spermatogenic wave.
\end{abstract}

\section{Introduction}

Normal fertility in males of most heterogametic species (i.e., species in which either males or females have nonidentical sex chromosomes; e.g., in humans where males have an $\mathrm{X}$ and a $\mathrm{Y}$ chromosome) requires the constant production of sperm over a long time period; in humans, production begins at puberty and usually continues until death. Spermatogenesis is a very complex, highly organized and regulated process that takes place in the seminiferous epithelium of testis tubules (Figure 1) and involves three major fundamental biological processes: the renewal of stem cells and the production and expansion of progenitor cells (mitosis); the reduction, by one-half, of the number of chromosomes in each progenitor cell (meiosis); and the unique differentiation of haploid cells (spermiogenesis) (Figure 1). In humans, each of these processes initiates at puberty and continues throughout life. Early progenitor cells, which are designated A spermatogonia in the mouse and A-darkspermatogonia in humans, are defined as "undifferentiated." These cells have the potential to become gametes but have not yet committed to the process. Undifferentiated spermatogonia divide mitotically to both repopulate the testicular stem cell population and provide progenitor cells that undergo spermatogenesis. Once spermatogonia enter the "differentiation" pathway, they become known as A1 spermatogonia in the mouse and A-palespermatogonia in humans and begin a series of irreversible differentiation steps leading to meiosis and spermiogenesis (1) (Figure 1). Differentiating spermatogonia in mice undergo five mitotic divisions before converting to preleptotene spermatocytes. This conversion represents the initiation of meiosis. From this point forward, the steps and cell types of spermatogenesis are conserved between mice and humans. Meiosis occurs in spermatocytes, and these cells can be divided into different subpopulations based on their chromatin structures (2). Recombination and separation of homologous chromosomes occurs in pachytene spermatocytes during meiosis I and results in the formation of secondary spermatocytes. These cells then proceed through meiosis II, wherein sister chromatids are separated into individual cells. At the completion of meiosis, four haploid gametes, termed round spermatids, result from the division of every spermatocyte. Each round spermatid then undergoes dramatic changes in its cellular morphology (spermiogenesis) to form first an elongating spermatid and finally a spermatozoon.

The seminiferous epithelium comprises Sertoli cells and germ cells and is surrounded by a layer of peritubular myoid cells, which

Conflict of interest: The authors have declared that no conflict of interest exists. Citation for this article: J Clin Invest. 2010;120(4):956-962. doi:10.1172/JCI41303. provide structural support. Sertoli cells maintain the integrity of the epithelium and support spermatogenesis through cell-cell contact with the germ cells (3). At the completion of spermiogenesis, immature spermatozoa are released from the seminiferous epithelium into the tubule lumen. Sertoli cells facilitate this process by helping degrade the cell-cell junctions binding them to late-stage spermatids (4), resulting in a progressive loss of contact between the two cell types. From the testis tubules, immature spermatozoa passively flow into the epididymis via the rete testis (Figure 1). Within the epididymis, spermatozoa acquire motility and the potential to fertilize an oocyte.

The endocrine regulation of spermatogenesis occurs through interplay among gonadotropins, steroids, and the somatic cells of the testis (i.e., Sertoli cells and Leydig cells) and through effects of vitamin A. Leydig cells, which are found between the seminiferous tubules, respond to luteinizing hormone (LH) from the pituitary gland and synthesize the male steroid hormone testosterone (2). Sertoli cells are regulated by follicle-stimulating hormone (FSH) from the pituitary gland and testosterone from the Leydig cells. In the past few years, the roles of FSH and testosterone and their actions on Sertoli cells and ultimately spermatogenesis have become more apparent, because of the use of directed gene deletions in mice (5-7). In contrast, the essential role of vitamin A (in the form of retinol or retinoic acid [RA]) with respect to spermatogenesis is just beginning to be appreciated. Vitamin A is required for fertility and normal spermatogenesis, and recently the mechanisms that drive RA regulation of germ cell development have begun to be understood. Here, we present a brief summary of how the organization and cyclic differentiation of germ cells in the testis leads to the continual production of sperm and how the actions of FSH and testosterone on Sertoli cells control spermatogenesis. The main focus of this Review is RA regulation of germ cell development, and we discuss and merge old and new data to present a new summary of why we believe that vitamin A is critical for normal and continual sperm production.

\section{Organization of spermatogenesis in time and space}

The seminiferous epithelium of most male mammals appears highly organized. Well-defined groups of germ cells appear along the tubule sequentially or at a single points on the tubule at different times (8). These defined groups of germ cells, termed stages (also known as cell associations), are morphologically distinct and can be identified in histological testis tubule cross sections such as the one pictured in Figure 1B. Stages are often depicted in charts showing the types of associations that are unique for a particular 


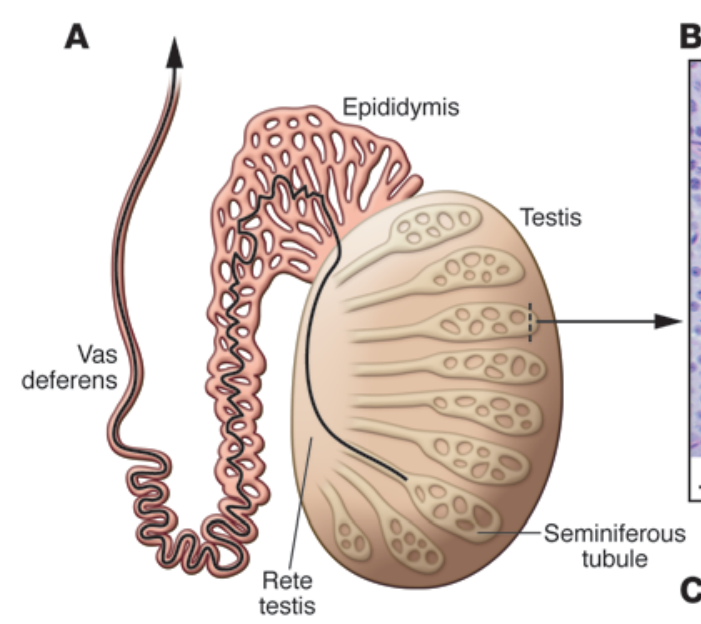

B Histological cross section of mouse tesis

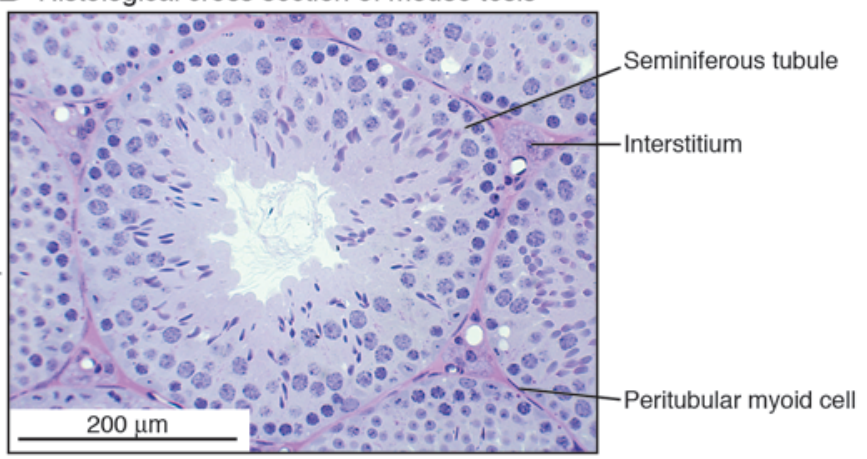

C Regulation of cellular differentiation in testis

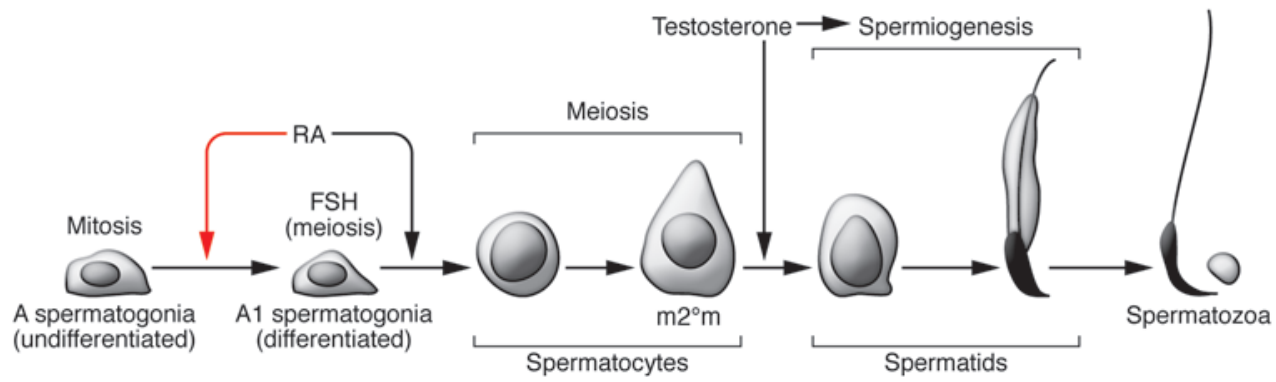

\section{Figure 1}

The structure, regulation, and cell types of the mouse testis. (A) The mammalian testis is composed of seminiferous tubules intertwined so that the "start" and "end" of these tubules are both connected to the rete testis. Immotile sperm flow from the lumen of the seminiferous tubules into the epididymis via the rete testis. During their passage through the epididymis to the vas deferens, sperm acquire their motility. Adapted with permission from Nature Reviews Genetics (59). (B) Histological cross section through an adult mouse testis depicting seminiferous tubules, the peritubular myoid cells, and the interstitium (space between tubules). (C) Expansion of both the undifferentiated (A spermatogonia) and differentiated (A1 spermatogonia) spermatogonial populations occurs by mitosis of these cell types, regulated in part by FSH. Undifferentiated spermatogonia enter the differentiation pathway at the time of the A-to-A1 spermatogonia transition. The red arrow denotes the required action of vitamin $\mathrm{A}$ (in the form of $\mathrm{RA}$ ) in this transition. The subsequent conversion of differentiated spermatogonia to spermatocytes, representing the initiation of meiosis, also requires RA activity. The differentiation of secondary spermatocytes $\left(\mathrm{m} 2^{\circ} \mathrm{m}\right)$ and the process of spermiogenesis (round spermatids to elongated spermatids, then spermatozoa) requires testosterone.

species (Figure 2A). For example, there are 12 distinct stages in the mouse, and each stage is represented by a specific set of germ cells being present at a single point on the tubule at the same time. There are six such stages in the human (9). When adult mouse testis tubules are examined in cross section, each tubule visible in the cross section can be assigned to a particular stage, depending on the germ cells present in that tubule. However, if a tubule is analyzed longitudinally, one can see that these stages are arranged such that the germ cells in association naturally progress to the next phase of their development, or transition to the next stage. The progression of germ cells through each stage along a tubule is known as the spermatogenic wave. If we were able to observe germ cell differentiation over time at a single point on the tubule, we would see the appearance of a defined group of cell associations followed by several others and then the reappearance of the original set of associations, constituting the cycle of the seminiferous epithelium. In any given mammal, the number of stages is arbitrary (i.e., makes no reference to germ cell development) and specific to the species. These numbered staging schemes are the widely accepted system for describing spermatogenesis. A full description of different mammalian staging schemes is beyond the scope of this review; however, a list of these can be found in ref. 2.
In reality, germ cell differentiation is a continuum. For many students of spermatogenesis, the description and depiction of the cycle results in confusion or, at best, indifference. However, there is a very simple way to understand the cycle and how it is generated. First, one must assume that the timing of the progression of germ cell development is intrinsic to the germ cells of a given species and not affected by somatic cells. This assumption comes from studies in which rat germ cells were transplanted into mouse testes. The timing of germ cell development and the subsequent cellular associations were those of the donor rat germ cells and not of the mouse recipient (10). At a single point along a mouse seminiferous tubule, the transition of undifferentiated A spermatogonia into the differentiation pathway (i.e, their transition to A1 spermatogonia) and therefore into meiosis occurs every 8.6 days (Figure $2 \mathrm{~B})$. The timing of this transition also coincides with the release of spermatozoa into the tubule lumen at that single point on the tubule. Thus, every 8.6 days in the mouse (16 days in humans), at any given point along a tubule, spermatogonia will differentiate at the base of the tubule and spermatozoa will be released into the lumen. In the mouse, these two processes both occur during stage VIII. In humans the differentiation of A-palespermatogonia appears to take place over stages II-VI, whereas spermatozoa are released 
A
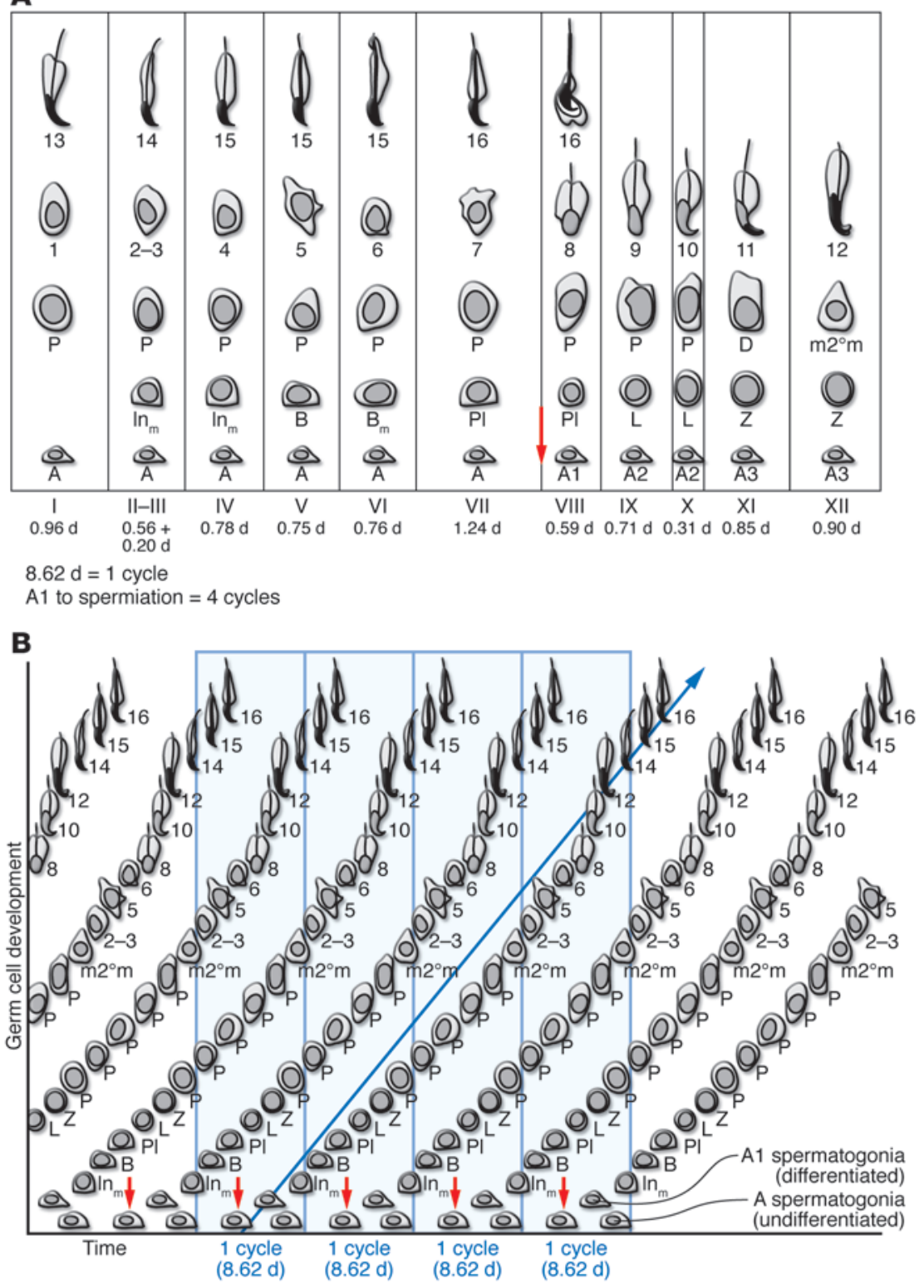

\section{Figure 2}

The cycle of the seminiferous epithelium. (A) Standard depiction of the cycle of the seminiferous epithelium for the mouse testis. The variable distances between the stages of the cycle are proportional to the duration of each of these cellular associations. The red arrow indicates the time in the cycle when vitamin $A$ is required for the commitment to meiosis. (B) Depiction of how the cycle is generated. Spermatogonia undergo mitotic expansion, and as a result of the action of vitamin A (in the form of RA) (red arrows), they initiate meiosis and ultimately become spermatozoa. The time required for this process from the time of the onset of meiosis to the formation of spermatozoa is particular to the species and the germ cells themselves (blue arrow). The periodic initiation of the differentiation process by vitamin A generates the cellular associations that define the cycle in $\mathbf{A}$. $\mathrm{In}_{\mathrm{m}}$, intermediate (mitosis); B, B spermatogonia; $\mathrm{PI}$, preleptotene spermatocytes; L, leptotene spermatocytes; Z, zygotene spermatocytes; $P$, pachytene spermatocytes; $D$, diplotene spermatocytes; $\mathrm{m} 2^{\circ} \mathrm{m}$, secondary spermatocytes. Round and elongating spermatids are labeled as steps $2-3,8,12,16$. at the very beginning of stage III (9). Nevertheless, given that mice and humans possess similar cell types and that a cycle of germ cell development is present in both species, the mouse is an excellent model for the study of mammalian spermatogenesis.

It is clear from Figure $2 \mathrm{~B}$ that the temporally controlled entry of spermatogonia into the differentiation pathway generates the cycle of the seminiferous epithelium. The net result of the cycle in the mouse is that spermatozoa are released at any given point along the tubule every 8.6 days. Moving along the tubule in space, it becomes apparent that the stages of the cycle appear in an orderly sequence, that is, adjacent stages appear next to one another (Figure 3). This phenomenon, the spermatogenic wave, is essentially the changing cellular associations in space instead of time $(3,11)$. The wave can be generated by the phasing along the tubule of the temporally controlled entry of germ cells into the differentiation pathway. The net results of the wave are the asynchronous phased release of spermatozoa at stage VIII of the cycle and, when this occurs along the entire length of the tubule, the continual production of spermatozoa.

\section{Sertoli cells, FSH, and testosterone}

It is well accepted that the hypothalamus acts to control fertility through the release of gonadotropin-releasing hormone (GnRH). This hormone acts on the pituitary gland to stimulate the secretion of two products critical for germ cell development in both males and females, FSH and LH (12). LH is responsible for triggering testicular Leydig cells to produce and secrete testosterone, and both FSH and testosterone exert their actions, which are critical for the normal progression of spermatogenesis, directly on Sertoli cells via their receptors, FSH receptor (FSHR) and androgen receptor (AR), respectively (8). Testosterone can also indirectly affect Sertoli cell function via signaling through the peritubular myoid cells (13, 14). Therefore, mutations in the genes encoding these hormones or their receptors have repercussions for normal sperm production. 


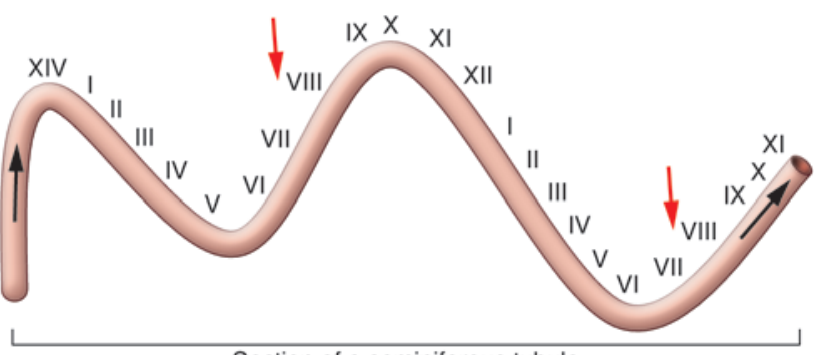

Section of a seminiferous tubule

Figure 3

Depiction of the spermatogenic wave. A single seminiferous tubule is depicted, and the stages of the cycle (cellular associations) along the tubule are shown. The spermatogenic wave describes the process in space, while the cycle of the seminiferous epithelium refers to the process in time. The point of meiotic initiation (red arrows) moves along the tubule in the direction of the black arrows. The net result of the wave is the asynchronous (and therefore continual) release of spermatozoa.

The study of mice and humans with null mutations in the genes encoding the $\beta$-subunit of FSH (FSH $\beta$ ) and FSHR has provided evidence to suggest that FSH is not essential for spermatogenesis but is required for quantitatively normal sperm production in both mice and humans $(5,15-18)$. Mice lacking Fshb exhibit reduced testis size, sperm count, and sperm motility; however, all stages of phenotypically normal spermatogenesis were identified among some of the tubules of these mice (16). Test matings with $F s h b$-knockout male mice produced normal litter sizes, whereas natural mutations in the human FSHB gene lead to azoospermia, reduced testis size, and infertility (19). The observed fertility difference between mice and men lacking FSH $\beta$ could be the result of differing genetic backgrounds; knockout mice are usually generated on highly inbred strains, whereas the human genetic background is much more diverse. Fshr-knockout male mice, like the Fshb-null males, are fertile, and their small testis size is attributed to a reduction in Sertoli cell numbers $(17,18)$. In addition, men carrying mutations in the FSHR gene have reduced sperm counts, impaired sperm motility, and low testicular volume (20) but can father children. Taken together, these studies suggest that FSH is important for determining Sertoli cell numbers in both species. Due to their close association with the developing germ cells, Sertoli cell numbers are directly linked to the number of sperm that can be produced; each Sertoli cell can only support a finite number of germ cells. Therefore, a lack of circulating FSH or an inability of Sertoli cells to respond to FSH, as is the case in men carrying mutations in the FSHR gene, leads to a reduction in sperm count because there are not enough Sertoli cells to support the maximum number of developing germ cells. While FSH may not be absolutely necessary for normal sperm production in male mice and humans, it is very important for species in which propagation of an individual depends on competitive numbers of sperm. For example, female chimpanzees are mated by several males; therefore, the male with the highest quality sperm and/or largest sperm count has the best chance of passing on his genome to the next generation. It may also play an important role in seasonal breeders where testicular activity is cyclic (21).

The action of testosterone on spermatogenesis can also be mediated through Sertoli cells (22). Although mouse models for complete androgen insensitivity, such as the testicular feminized mutant (tfm) mouse, have been available for many years, two different research groups used Cre-Lox technology to produce mice with a Sertoli cell-specific knockout of the Ar gene (referred to herein as SCARKO mice) $(7,23-25)$. The phenotypes seen in both tfm mutant and SCARKO male mice are highly conserved. Although the gene knockout procedures were somewhat different in the two SCARKO studies $(7,23,25)$, the results were similar. Progression of late-round spermatids to elongating spermatids was sensitive to the loss of Sertoli cell AR function, but spermatocyte development and progression through meiosis were surprisingly unaffected. Stereological analysis in one of these studies indicated that Sertoli cell numbers were unchanged in SCARKO mice, but spermatocyte, round spermatid, and elongated spermatid numbers were reduced to $64 \%, 3 \%$, and $0 \%$, respectively, when compared with their numbers in WT mice (25). When SCARKO mice lacking FSHR were analyzed, germ cell numbers were reduced to $20 \%$ of the numbers in SCARKO mice, as a result of failure to progress beyond early meiosis (26). The results from these studies confirm that spermatogenesis requires the action of both FSH and androgen on Sertoli cells, but the initial entry of germ cells into meiosis seems to be independent of direct action of either hormone. Androgen action in the testis does, however, appear to regulate postmeiotic differentiation. There is no evidence to suggest that either FSH or testosterone in excess or deficiency affects the duration of the cycle of the seminiferous epithelium or the spermatogenic wave.

\section{RA initiates meiosis and may generate the cycle of the seminiferous epithelium and the spermatogenic wave}

While the requirement of FSH and androgens for spermatogenesis is well established, the actions of RA on this process are equally important but less well characterized. Previous studies on vitamin A-deficient rodents (27-31), combined with more recent studies (32-34) on the induction of stimulated by retinoic acid gene 8 (STRA8) protein, suggest that the key control over meiotic initiation is vitamin A in the form of RA. The results also imply a role for vitamin $A$ in the formation of the cycle of the seminiferous epithelium and the spermatogenic wave, although definitive proof of this requires further experimentation. The general transport and metabolism of vitamin A and evidence for its participation in the onset of meiosis and the cycle of the seminiferous epithelium are summarized below.

The transport and metabolism of vitamin A. In general, the actions of vitamin $\mathrm{A}$ in the testis are mediated by transport, metabolism, and degradation of retinoids (compounds related chemically to vitamin A) and activation of its nuclear receptors (35) (Figure 4). As RA is poorly transported via serum, oxidation of dietary vitamin A (retinol) to RA generally takes place in target tissues. Retinol is stored in the liver and transported to target tissues (36) that express the required intracellular binding proteins and retinoid metabolism enzymes so that the production and degradation of RA can be tightly regulated (37). Chung and Wolgemuth (38) and Vernet et al. (39) examined the expression patterns of some of the retinoid metabolism enzymes and how their function can affect spermatogenesis and fertility. Within cells, RA binds to two families of intracellular receptors termed RA receptors (RARs), which bind all-trans and 9-cis RA, and retinoid X receptors (RXRs), which bind 9-cis RA $(35,40)$. These receptors, in the form of homo- and heterodimers, regulate gene expression by binding to specific elements in the promoter regions of genes under the control of vitamin A. While many of the isoforms of RAR and RXR are found in various testis cell types at different developmental 


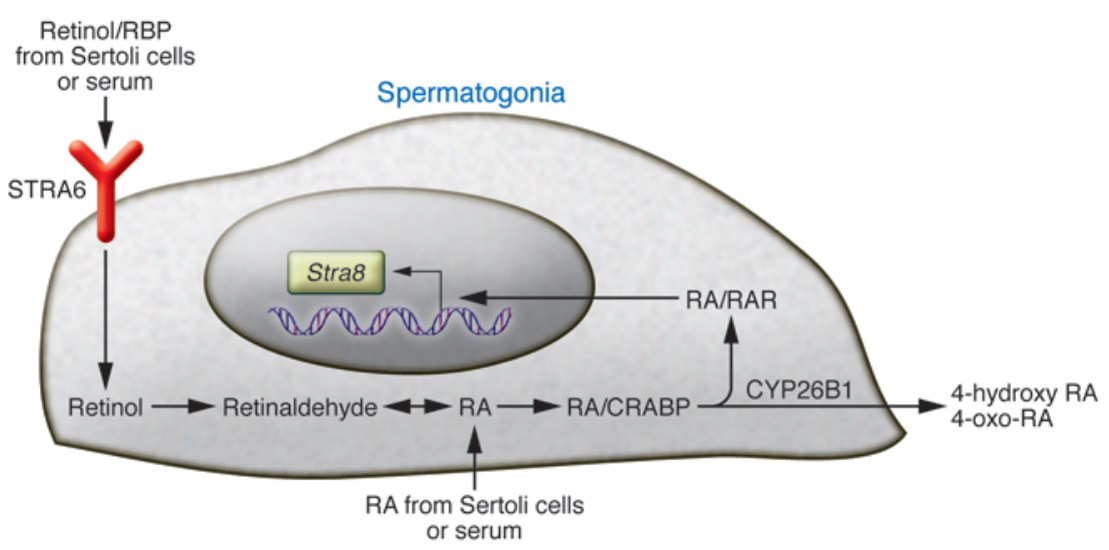

Figure 4

The fate of vitamin A in the spermatogonia. Retinol is delivered to germinal cells on the retinol-binding protein (RBP) and is internalized via the membrane receptor STRA6. It is also plausible that RA is delivered directly to spermatogonia either by Sertoli cells or from the serum. Inside the cell, retinol is converted to RA in a two-step process and can interact with RARs such as RAR $\gamma$. The RA is bound up by an excess of cellular retinoic acid-binding protein (CRABP). The activated receptor can stimulate transcription of a number of genes including Stra8, which has been shown to be necessary for progression through meiosis. Excess RA can be metabolized by the enzyme CYP26B1 into 4-oxo and 4-hydroxy forms. These forms are then secreted from the cell.

stages, there seems to be a general consensus that RA mediates its effects primarily via the action of RAR $\alpha$ in Sertoli cells and via the action of RAR $\gamma$ in early germ cells. Rara-knockout males are sterile, and transplantation experiments demonstrated that germline stem cells carrying mutant Rara alleles that colonized a germ cell-depleted testis were able to progress through spermatogenesis normally (41). This suggests that Sertoli cell expression of RAR $\alpha$ is critical for normal spermatogenesis. The precise mechanism by which RAR $\alpha$ signaling exerts its effects in Sertoli cells is not yet understood. Deletion of Rxrb in male mice leads to a failure of spermatid release and testis degeneration (42); therefore, it is clear that regulating the expression of RA receptors is important for mammalian spermatogenesis and fertility.

In many ways, the liver and Sertoli cells appear to function similarly in the uptake of retinol and the maintenance of large pools of this retinoid $(35,43)$. However, it seems that spermatids and testicular and epididymal sperm also store retinoids (44). Sertoli cells have been reported to be the main site of RA synthesis in the testis, and it has been postulated that Sertoli cells "distribute" RA to germ cells (35). Sertoli cells could also deliver retinol to germ cells or spermatogonia could access retinol and RA directly from serum via the vascular system $(35,39)$. There is evidence to suggest that the delivery of RA to germ cells is tightly regulated and that different germ cells can respond to RA in distinct ways; RA appears to be responsible for the differentiation of undifferentiated spermatogonia, the initiation of meiosis, and possibly the initiation of the cycle of the seminiferous epithelium. In the following sections, we discuss current evidence to support a role for RA in each of these processes, provide a brief summary of the delivery of RA to germ cells, and outline our limited understanding of what is known about RA regulation of human spermatogenesis.

$R A$ regulation of spermatogonial differentiation and meiotic initiation in rodents. A role for vitamin A in mammalian spermatogonial differentiation and meiotic initiation is beginning to unfold. It has been known since 1925 that vitamin A is required for normal spermatogenesis (for a recent review of this subject, see ref. 35), as when male rodents are made vitamin A deficient, spermatogenesis ceases. Examination of vitamin A-deficient rodent testes reveals only undifferentiated spermatogonia and Sertoli cells within the seminiferous epithelium (27-31). Therefore, a lack of vitamin A leads to a block in the ability of spermatogonia to differentiate. In the testes of vitamin A-deficient rats, there are also some preleptotene spermatocytes present (45), so the block in spermatogonial differentiation is not as complete in rats as it is in mice. Recent in vitro culture experiments have shown that RA can induce spermatogonial differentiation (33). RA treatment of germline stem cells isolated from 2 days postpartum (dpp) male mice stimulated expression of $c$-kit, a well-characterized marker of spermatogonial differentiation.

In the past few years, the mechanism by which RA controls meiotic entry has been investigated. Data from mouse expression arrays showed that Stra8 transcript was at very high levels only in the ovary at E14.5 and in the neonatal postnatal testis during the progression of spermatogonial differentiation and entry into meiosis $(32,46-49)$. Stra8 was first described as one of a group of RA-responsive genes, and the full-length cDNA was isolated and sequenced from the mouse embryonic carcinoma cell line P19 (50). RA was shown to be necessary for the induction of Stra 8 in both ovaries and testes (46), and deletion of Stra 8 resulted in the prevention of meiosis in germ cells of both sexes $(32,51)$. RA appears to be present in both male and female embryonic urogenital ridges, but its action and the subsequent entry of gonocytes into meiosis in the embryonic male is inhibited by the presence of the enzyme cytochrome P450, family 26 , subfamily b, polypeptide 1 (CYP26B1) $(46,49)$. CYP26B1 degrades RA into metabolites, some of which are inactive. If CYP26B1 is inhibited in mouse embryonic testes in culture or if the gene encoding CYP26B1 is ablated, Stra8 mRNA is synthesized in male mouse germ cells and meiosis is initiated $(46,49,52)$. Induction of STRA8 in embryonic male germ cells is sufficient to induce the synthesis of downstream markers of meiosis, such as synaptonemal complex protein 3 (SCP3) and the meiosis-specific recombinase DMC1 (49). Therefore, the expression of Stra 8 is necessary for germ cells to enter into meiosis and is an excellent marker for the action of RA.

Until recently, studies on the action of retinoids on the adult mouse testis have focused on Sertoli cells. Since both FSH and androgens act on Sertoli cells and since Sertoli cells have RARs and other components of the vitamin A response, this focus was reasonable. Still, whether retinoids act directly on germ cells, which also express RARs, or whether their actions are indirectly mediated through Sertoli cells or peritubular myoid cells remained unknown until recently. It was determined that Stra 8 expression in the adult mouse testis and in isolated germ cells could be induced by RA both in vivo and in culture $(33,34)$; therefore, germ cells can directly respond to RA stimulation. In developmental studies, the peak of Stra 8 mRNA expression in the developing mouse testis coincides temporally with the onset of meiosis (47). In normal 
mouse testes, STRA8 protein is detected in gonocytes as early as $5 \mathrm{dpp}$, and in the adult mouse testes, the highest levels of Stra 8 mRNA and protein are found in seminiferous tubules in stages VI-VIII of the cycle of the seminiferous epithelium (34). It is during these stages that A-to-A1 spermatogonial differentiation occurs and that preleptotene spermatocytes are formed, representing the initiation of meiosis. It is clear that RA is important for both these processes to occur normally. Thus, it appears as though RA regulation of spermatogonial differentiation and the onset of meiosis is driven by the action of RA directly on germ cells.

$R A$ and the cycle of the seminiferous epithelium. Current evidence to support a role for RA in the establishment of the mammalian cycle of the seminiferous epithelium is derived from analysis of vitamin Adeficient rodents. When retinol is given back to these animals, spermatogenesis is reinitiated by stimulating A-to-A1 spermatogonial differentiation in a synchronized manner throughout the entire testis. The spermatogenic wave disappears, and only closely related stages can be found in tubules across a histological cross section of these testes (31). This synchronization is maintained for many months. In addition, in mice, this results in the release of spermatozoa only every 8.6 days; therefore, the vitamin A-driven synchronization in the mouse testis eliminates continual sperm production. The task for researchers is now to determine whether the tightly regulated delivery of RA at specific points along the tubule controls the spermatogenic wave and the cycle of the seminiferous epithelium. It is plausible that the spermatogenic wave is established at the onset of spermatogenesis by the progressive development of cells that are responsive to vitamin A along the tubule; however, this has yet to be investigated. What is known is that the deficiency and then repletion of vitamin A synchronizes spermatogonial differentiation and meiotic entry. In addition, the asynchronous production of spermatozoa is disrupted by vitamin A deficiency.

Making $R A$ available to germ cells. In normal animals, the availability of RA to germ cells is under tight control. In vitamin A-sufficient adult male mice, exogenous RA but not retinol, administered by systemic injection, stimulates Stra 8 mRNA expression in the testis (34). Exogenous RA also increases the number of preleptotene spermatocytes incorporating 5-bromo-2-deoxyuridine, indicating a more synchronized premeiotic DNA replication (34). It is likely that the in vivo administration of retinol is ineffective in the induction of Stra 8 mRNA because it is converted to retinyl esters in Sertoli cells and enters the vitamin A storage pools while RA goes directly (but inefficiently) to the A spermatogonia. However, the ability to push germ cells into the differentiation pathway or meiosis by administration of exogenous RA reveals that RA availability is limiting and carefully controlled in the normal mouse testis. Thus, the differential activity of RA in stimulating meiosis, and possibly the cycle of the seminiferous epithelium and the spermatogenic wave, appears to result from the control of RA levels.

$R A$ regulation of buman spermatogenesis. The current understanding of RA function in human spermatogenesis is extremely limited. Vitamin A deficiency does occur in humans and is one of the leading causes of blindness, especially in developing countries (53); however, there is no information regarding the reproductive capabilities of these people. There is only minimal information available regarding the effect of RA on human male germ cells and the expression of RA metabolism enzymes in men. Germ cells in fetal human testes cultured with RA were found to undergo apoptosis after they were stimulated to proliferate (54), a result similar to that seen in cultures of fetal mouse testes (55). Human Sertoli cells express different enzymes of the vitamin A metabolism pathway $(36,56)$, as is seen in the mouse, and STRA8 is expressed in the human embryonic ovary and testis (57) and the postnatal testis (58). Clearly there are large gaps in our knowledge with respect to RA regulation of human spermatogenesis; however, the rodent studies outlined in this Review and the high level of cell type conservation in the testis between humans and rodents imply that vitamin A could very well be critical in humans for spermatogonial differentiation, meiosis, and the cycle of the seminiferous epithelium.

\section{Conclusions}

The combined actions of FSH, testosterone, and RA are essential for normal mammalian spermatogenesis. FSH acts on Sertoli cells and may affect spermatogonial populations. RA acts on both Sertoli cells and germ cells and pushes undifferentiated spermatogonia into the differentiation pathway and, eventually, meiotic prophase. Testosterone also acts on Sertoli cells and is necessary for round spermatid formation. While much has been learned from rodent models and directed gene knockouts, there are examples of human pathologies associated with inactivating mutations of FSHR and androgen insensitivity syndromes that have also taught us much, as they lead to compromised reproductive capacity. Vitamin A deficiencies that lead to a variety of human illnesses occur in many parts of the world, but there is a paucity of information regarding their effect on human reproductive capacities (53). Given the complexities associated with retinoid storage, transport, and uptake; the number of elements in the retinoid signaling pathway; the multiple intracellular receptors; and the control of the overall organization of spermatogenesis, the potential for male fertility pathologies associated vitamin A activity is large. However, given the importance of vitamin A in nearly every other organ system, it is likely that there is a great deal of genetic redundancy designed to protect the organism. Looking forward, the focus of future research is to determine the precise mechanism of retinoid metabolism within the testis - not an easy task given the multitude of different enzymes that could regulate this process. There is also much to be learned about whether RA is as critical to human spermatogenesis as it is in mice, and elaboration of the testicular RA production pathway in mice could provide screening targets for natural cases of human infertility.

\section{Acknowledgments}

Work in the authors' laboratory was supported by grants from $\mathrm{NIH}$ (HD 10808-33 and U54 42454). Appreciation is extended to Chris Small for critical reading of this manuscript.

Address correspondence to: Michael D. Griswold, School of Molecular Biosciences, Washington State University, Pullman, WA 99164-7520. Phone: 509.335.6281; Fax: 509.335.9688; E-mail: mgriswold@wsu.edu.
1. de Rooij DG, Russell LD. All you wanted to know
about spermatogonia but were afraid to ask.
JAndrol. 2000;21(6):776-798.
2. Russell LD, Ettlin, RA, Sinha Hikim AD, Clegg EP.
Histological and histopathological evaluation of the testis.
Clearwater, FL: Cache River Press; 1990.

3. Kerr JB, Loveland KL, O’Bryan MK, deKretser DM. Cytology of the testis and intrinsic control mechanisms. In: Neill JD, ed. Physiology of reproduction. New York, NY: Elsevier; 2006:827-947.
4. Yan HH, Mruk DD, Cheng CY. Junction restructuring and spermatogenesis: the biology, regulation, and implication in male contraceptive development. Curr Top Dev Biol. 2008;80:57-92.

5 . Kumar TR, et al. Transgenic models to study gonad- 
otropin function: the role of follicle-stimulating hormone in gonadal growth and tumorigenesis. Mol Endocrinol. 1999;13(6):851-865

6. Kumar TR, Wang Y, Lu N, Matzuk MM. Follicle stimulating hormone is required for ovarian follicle maturation but not male fertility. Nat Genet. 1997; 15(2):201-204.

7. Holdcraft RW, Braun RE. Androgen receptor function is required in Sertoli cells for the terminal differentiation of haploid spermatids. Development. 2004; 131(2):459-467.

8. Clermont Y. Kinetics of spermatogenesis in mammals: seminiferous epithelium cycle and spermatogonial renewal. Physiol Rev. 1972;52(1):198-236.

9. Amann RP. The cycle of the seminiferous epithelium in humans: a need to revisit? J Androl. 2008; 29(5):469-487.

10. Franca LR, Ogawa T, Avarbock MR, Brinster RL, Russell LD. Germ cell genotype controls cell cycle during spermatogenesis in the rat. Biol Reprod. 1998; 59(6):1371-1377.

11. Perey B, Clermont Y, LeBlond GP. The wave of the seminiferous epithelium in the rat. AmJ Anat. 1961; 108:47-77.

12. O'Shaughnessy PJ, Morris ID, Huhtaniemi I, Baker PJ, Abel MH. Role of androgen and gonadotrophins in the development and function of the Sertoli cells and Leydig cells: data from mutant and genetically modified mice. Mol Cell Endocrinol. 2009; $306(1-2): 2-8$.

13. Zhang $\mathrm{C}$, et al. Oligozoospermia with normal fertility in male mice lacking the androgen receptor in testis peritubular myoid cells. Proc Natl Acad SciUS A. 2006;103(47):17718-17723.

14. Welsh M, Saunders PT, Atanassova N, Sharpe RM, Smith LB. Androgen action via testicular peritubular myoid cells is essential for male fertility. FASEBJ 2009;23(12):4218-4230.

15. Vaskivuo TE, et al. Effects of follicle-stimulating hormone (FSH) and human chorionic gonadotropin in individuals with an inactivating mutation of the FSH receptor. Fertil Steril. 2002;78(1):108-113.

16. Kumar TR, Wang Y, Lu N, Matzuk MM. Follicle stimulating hormone is required for ovarian follicle maturation but not male fertility. Nat Genet. 1997; 15(2):201-204

17. Dierich A, et al. Impairing follicle-stimulating hormone (FSH) signaling in vivo: targeted disruption of the FSH receptor leads to aberrant gametogenesis and hormonal imbalance. Proc Natl Acad SciUS A. 1998;95(23):13612-13617.

18. Abel MH, Wootton AN, Wilkins V, Huhtaniemi I Knight PG, Charlton HM. The effect of a null mutation in the follicle-stimulating hormone receptor gene on mouse reproduction. Endocrinology. 2000; 141(5):1795-1803.

19. Layman LC, McDonough PG. Mutations of follicle stimulating hormone-beta and its receptor in human and mouse: genotype/phenotype. Mol Cell Endocrinol. 2000;161(1-2):9-17.

20. Tapanainen JS, Aittomaki K, Min J, Vaskivuo T, Huhtaniemi IT. Men homozygous for an inactivating mutation of the follicle-stimulating hormone (FSH) receptor gene present variable suppression of spermatogenesis and fertility. Nat Genet. 1997 15(2):205-206.

21. Bartke A, Amador AG, Chandrashekar V, Klemcke HG. Seasonal differences in testicular receptors and steroidogenesis. J Steroid Biochem. 1987; 27(1-3):581-587.
22. Johnston DS, Russell LD, Friel PJ, Griswold MD. Murine germ cells do not require functional androgen receptors to complete spermatogenesis following spermatogonial stem cell transplantation. Endocrinology. 2001;142(6):2405-2408

23. Denolet E, et al. The effect of a sertoli cell-selective knockout of the androgen receptor on testicular gene expression in prepubertal mice. Mol Endocrinol. 2006;20(2):321-334.

24. Verhoeven G. A Sertoli cell-specific knock-out of the androgen receptor. Andrologia. 2005;37(6):207-208.

25. De Gendt K, et al. A Sertoli cell-selective knockout of the androgen receptor causes spermatogenic arrest in meiosis. Proc Natl Acad Sci U S A. 2004 101(5):1327-1332.

26. Abel MH, et al. Spermatogenesis and sertoli cell activity in mice lacking sertoli cell receptors for follicle-stimulating hormone and androgen. Endocrinology. 2008;149(7):3279-3285.

27. Mitranond V, Sobhon P, Tosukhowong P, Chindaduangrat W. Cytological changes in the testes of vitamin-A-deficient rats. I. Quantitation of germinal cells in the seminiferous tubules. Acta Anatomica. 1979;103(2):159-168.

28. Unni E, Rao MR, Ganguly J. Histological \& ultrastructural studies on the effect of vitamin A depletion \& subsequent repletion with vitamin A on germ cells \& Sertoli cells in rat testis. Indian J Exp Biol. 1983;21(4):180-192.

29. van Pelt AM, de Rooij DG. Synchronization of the seminiferous epithelium after vitamin A replacement in vitamin A-deficient mice. Biol Reprod. 1990;43(3):363-367.

30. McLean DJ, Russell LD, Griswold MD. Biological activity and enrichment of spermatogonial stem cells in vitamin A-deficient and hyperthermiaexposed testes from mice based on colonization following germ cell transplantation. Biol Reprod. 2002; 66(5):1374-1379.

31. Griswold MD, Bishop PD, Kim KH, Ping R, Siiteri JE, Morales C. Function of vitamin A in normal and synchronized seminiferous tubules. Ann NY Acad Sci. 1989;564:154-172.

32. Anderson EL, et al. Stra8 and its inducer, retinoic acid, regulate meiotic initiation in both spermatogenesis and oogenesis in mice. Proc Natl Acad Sci US A. 2008;105(39):14976-14980.

33. Zhou Q, et al. Expression of stimulated by retinoic acid gene 8 (Stra8) and maturation of murine gonocytes and spermatogonia induced by retinoic acid in vitro. Biol Reprod. 2008;78(3):537-545.

34. Zhou Q, et al. Expression of stimulated by retinoic acid gene 8 (Stra8) in spermatogenic cells induced by retinoic acid: an in vivo study in vitamin A-sufficient postnatal murine testes. Biol Reprod. 2008; $79(1): 35-42$.

35. Livera G, Rouiller-Fabre V, Pairault C, Levacher C, Habert R. Regulation and perturbation of testicular functions by vitamin A. Reproduction. 2002; 124(2):173-180.

36. Paik J, et al. Vitamin A: overlapping delivery pathways to tissues from the circulation. J Nutr. 2004; 134(4):276S-280S.

37. Molotkov A, Ghyselinck NB, Chambon P, Duester G. Opposing actions of cellular retinol-binding protein and alcohol dehydrogenase control the balance between retinol storage and degradation. Biochem J. 2004;383(pt 2):295-302.

38. Chung SS, Wolgemuth DJ. Role of retinoid signaling in the regulation of spermatogenesis. Cytogenet
Genome Res. 2004;105(2-4):189-202

39. Vernet $N$, et al. Retinoic acid metabolism and signaling pathways in the adult and developing mouse testis. Endocrinology. 2006;147(1):96-110.

40. Mark M, et al. A genetic dissection of the retinoid signalling pathway in the mouse. Proc Nutr Soc. 1999; 58(3):609-613.

41. Doyle TJ, Braun KW, McLean DJ, Wright RW, Griswold MD, Kim KH. Potential functions of retinoic acid receptor A in Sertoli cells and germ cells during spermatogenesis. Ann N Y Acad Sci. 2007; 1120:114-130.

42. Kastner P, et al. Abnormal spermatogenesis in RXR beta mutant mice. Genes Dev. 1996;10(1):80-92.

43. Bishop PD, Griswold MD. Uptake and metabolism of retinol in cultured Sertoli cells: evidence for a kinetic model. Biochemistry. 1987;26(23):7511-7518.

44. Ren P, Bishop PD. Uptake and metabolism of retinol in isolated cells of germinal epithelium in vitro. JTongii Med Univ. 1989;9(1):36-43.

45. Huang HF, Hembree WC. Spermatogenic response to vitamin A in vitamin A deficient rats. Biol Reprod. 1979;21(4):891-904.

46. Koubova J, Menke DB, Zhou Q, Capel B, Griswold $\mathrm{MD}$, Page DC. Retinoic acid regulates sex-specific timing of meiotic initiation in mice. Proc Natl Acad SciU S A. 2006;103(8):2474-2479.

47. Shima JE, McLean DJ, McCarrey JR, Griswold MD. The murine testicular transcriptome: characterizing gene expression in the testis during the progression of spermatogenesis. Biol Reprod. 2004;71(1):319-330.

48. Small CL, Shima JE, Uzumcu M, Skinner MK, Griswold MD. Profiling gene expression during the differentiation and development of the murine embryonic gonad. Biol Reprod. 2005;72(2):492-501.

49. Bowles J, et al. Retinoid signalling determines cell fate in mice. Science. 2006;312(5773):596-600.

50. Oulad-Abdelghani M, et al. Characterization of a premeiotic germ cell-specific cytoplasmic protein encoded by Stra8, a novel retinoic acid-responsive gene. J Cell Biol. 1996;135(2):469-477.

51 . Baltus AE, et al. In germ cells of mouse embryonic ovaries, the decision to enter meiosis precedes premeiotic DNA replication. Nat Genet. 2006;38(12):1430-1434.

52. MacLean G, Li H, Metzger D, Chambon P, Petkovich M. Apoptotic extinction of germ cells in testes of Cyp26b1 knockout mice. Endocrinology. 2007; 148(10):4560-4567.

53. Sommer A. The continuing challenge of vitamin A deficiency. Ophthalmic Epidemiol. 2009;16(1):1.

54. Lambrot R, et al. Use of organ culture to study the human fetal testis development: effect of retinoic acid. J Clin Endocrinol Metab. 2006;91(7):2696-2703.

55. Trautmann E, Guerquin MJ, Duquenne C, Lahaye JB, Habert R, Livera G. Retinoic acid prevents germ cell mitotic arrest in mouse fetal testes. Cell Cycle. 2008; $7(5): 656-664$.

56. Lindqvist A, He YG, Andersson S. Cell type-specific expression of beta-carotene 9',10'-monooxygenase in human tissues. J Histochem Cytochem. 2005. 53(11):1403-1412.

57 . Houmard B, et al. Global gene expression in the human fetal testis and ovary. Biol Reprod. 2009; 81(2):438-443

58. Miyamoto T, et al. Isolation and expression analysis of the testis-specific gene, STRA8, stimulated by retinoic acid gene 8. J Assist Reprod Genet. 2002; 19(11):531-535.

59. Cooke HJ, Saunders PT. Mouse models of male infertility. Nat Rev Genet. 2002;3(10):790-801. 